\title{
Implications of prescribing a fixed-dose combination in clinical cardiology practice: a retrospective observational study using a single medical centre database in Korea
}

\author{
Hyungseop Kim, Hyuck-Jun Yoon, Hyoung-Seob Park, Yun-Kyeong Cho, \\ Chang-Wook Nam, Seongwook Han, Seung-Ho Hur, Yoon-Nyun Kim, Kwon-Bae Kim
}

\section{Division of}

Cardiology, Department of Internal Medicine, Keimyung University Dongsan Medical Center, Daegu, Republic of Korea

\section{Correspondence to} Dr Hyungseop Kim, Keimyung University Dongsan Medical Center,56 Dalseong-Ro, JungGu, Daegu 41931, Republic of Korea; khyungseop@dsmc.or.kr

Received 20 January 2017 Revised 22 May 2017 Accepted 24 May 2017

\section{CrossMark}

To cite: Kim H, Yoon $\mathrm{H}-\mathrm{J}$ Park H-S, et al. Heart Asia 2017:9:1-7.. doi:10.1136/ heartasia-2017-010885

\section{ABSTRACT}

Objective Fixed-dose combination (FDC) prescribing enhances adherence to medication. However, there are limited data regarding the usefulness of FDC drugs across different risk groups. The aim of this study was to explore the relationship between FDC discontinuation and clinical outcomes.

Methods From January 2008 to December 2014, patients with FDC prescriptions who visited a cardiology outpatient clinic at a tertiary university hospital in Daegu, Republic of Korea were retrospectively identified. The 10-year atherosclerotic cardiovascular disease (ASCVD) risk score and 20 conventional cardiovascular (CV) risk factors were assessed. Patients were classified according to FDC continuation, together with a tertile of 20 risks. $\mathrm{CV}$ events were defined as the composite of admission for worsening heart failure or diabetes, stroke, ischaemic heart disease, and CV death.

Results 502 patients were prescribed with one of the following FDC products: calcium channel blocker (CCB) plus angiotensin receptor blockers (ARB), CCB plus statins, and ARB plus diuretics. During follow-up (mean $2.8 \pm 2.4$ years), 203 discontinuations (40.4\%) occurred. FDC-discontinued patients had lower ASCVD risk scores ( $24.8 \%$ vs. $28.8 \%, p<0.001$ ), and patients with $<6$ risk factors discontinued FDC frequently. During follow-up, 57 events (11.4\%) were reported: 30 (14.8\%) in FDCdiscontinued patients and $27(9.1 \%)$ in FDC-continued patients $(p=0.062)$. In multivariate models accounting for events, FDC discontinuation $(p<0.001)$ and high ASCVD risk score $(p=0.017)$ were associated with CV events. Conclusions FDC discontinuation was common among patients attending the cardiology outpatient clinic. Our analyses suggest that FDC discontinuation in patients at high ASCVD risk may have an impact on CV event rates.

\section{INTRODUCTION}

Fixed-dose combination (FDC) products containing multiple drugs have been widely used and are available for the treatment of various cardiovascular (CV) disorders such as hypertension, hyperlipidaemia, ischaemic heart disease, and heart failure. FDC products are produced to simplify, easily administer, and enhance adherence to the prescription of multiple drugs. ${ }^{1-6}$

Regarding the prescribing of FDC drugs, most physicians put a premium on patient compliance, which can be enhanced by maintaining an FDC regimen. ${ }^{6-8}$ Because of this enhanced compliance, FDC can benefit patients as a result of the increased convenience, efficacy, and synergistic effects of lower doses of each drug component. ${ }^{9}$ Unfortunately, there are still some weaknesses associated with the use of FDC products, including titration limitation, lack of availability, and increased costs. ${ }^{10}$ Despite these disadvantages, a growing number of FDC formulations are in production today. $^{911}$

However, there are limited data or evidence regarding the definite benefits of FDC therapy in outpatient clinics. In addition, the consistency of FDC treatment across different risk groups is still unclear. Thus, the aim of this study was to explore which patients could adhere to and benefit from a continuous FDC regimen and to evaluate the relationship between the discontinuation of FDC and adverse CV outcomes.

\section{PATIENTS AND METHODS \\ Patients}

This is a retrospective observational study focusing on FDC administration. From January 2008 to December 2014, patients who visited the cardiology outpatient clinic of a single tertiary university hospital in Daegu, Republic of Korea, to evaluate either cardiac function or disorders and had received an FDC prescription, were identified via electronic medical records (EMR) (figure 1). The inclusion criteria were as follows: (1) age $>18$ years old; (2) FDC prescription provided at a cardiology outpatient clinic and not during admission; (3) the FDC drugs only included lipid- or blood pressure-lowering drugs (ie, no antiplatelet medications); and (4) there were available data on CV risk factors. Patients were excluded if: (1) they had received any earlier prescription of FDC medication during the baseline period in other hospitals; (2) non-CV FDC therapy was prescribed concomitantly; or (3) they had a malignant neoplasm or a psychiatric disorder. The index date was the first FDC prescribing date. The types of FDC included were restricted to three combinations of $\mathrm{CV}$ drugs: calcium channel blocker (CCB) plus angiotensin receptor blocker (ARB); $\mathrm{CCB}$ plus statins; and $\mathrm{ARB}$ plus diuretics. The study protocol conforms to the ethical guidelines of the Declaration of Helsinki. This research was approved by the institutional review board; written informed consent was waived because of the retrospective nature of this study. 


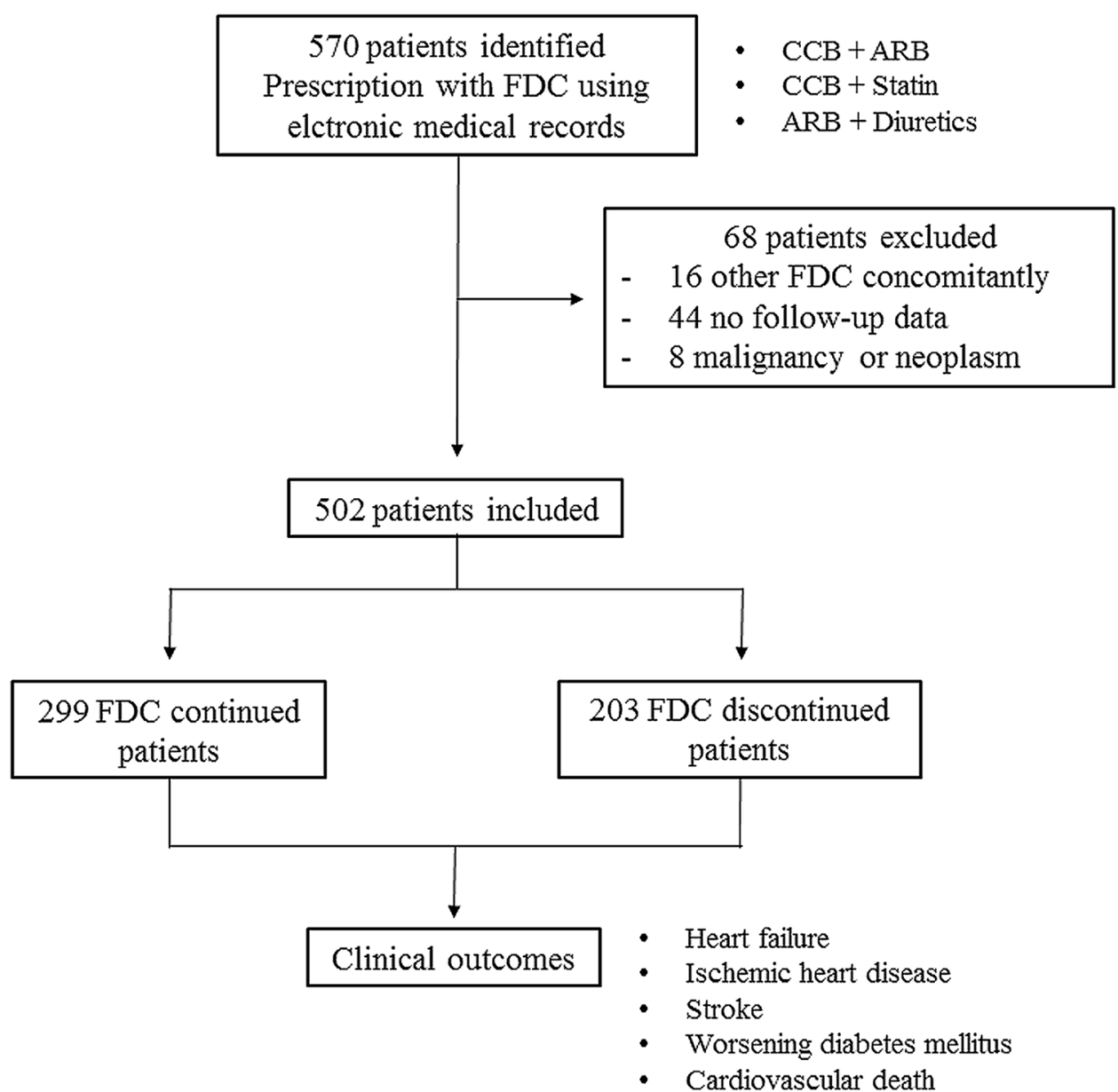

Figure 1 Study flow chart showing the number of participants in the study. FDC, fixed-dose combination; CCB, calcium channel blocker; ARB, angiotensin receptor blocker.

\section{Data acquisition and variables}

With thorough review of the EMR, 20 conventional $\mathrm{CV}$ risk factors were collected, and each risk factor was dichotomized with the following cut-off values: age ( $\geq 60$ years old), body mass index $\left(\geq 25 \mathrm{~kg} / \mathrm{m}^{2}\right)$, systolic blood pressure $(\geq 140 \mathrm{mmHg})$, total cholesterol $(\geq 200 \mathrm{mg} / \mathrm{dL})$, triglycerides $(\geq 150 \mathrm{mg} /$ $\mathrm{dL}$ ), high-density lipoprotein (HDL) cholesterol ( $\leq 45 \mathrm{mg}$ ) $\mathrm{dL}$ in men, $\leq 55 \mathrm{mg} / \mathrm{dL}$ in women), low-density lipoprotein (LDL) cholesterol $(\geq 150 \mathrm{mg} / \mathrm{dL})$, fasting glucose $(\geq 110 \mathrm{mg} /$ $\mathrm{dL})$, and creatinine $(\geq 1.1 \mathrm{mg} / \mathrm{dL})$. The following data were also used as risk factors: smoking and alcohol consumption; medical history of heart failure, dyslipidaemia, diabetes mellitus, hypertension, ischaemic heart disease, stroke, and peripheral vascular disease; family history of premature coronary heart disease $(<55$ years old in men, $<65$ in women); and chronic renal replacement therapy with dialysis or transplantation.

Furthermore, baseline CV risk score was assessed using the American College of Cardiology (ACC)/American Heart Association (AHA) atherosclerotic CV disease (ASCVD) algorithm to calculate the 10 -year risk of a CV event. In case the values of risk factors were outside the predefined range, the minimal or maximal value within each reference value range was entered.

\section{Follow-up and cardiovascular events}

The EMR were extracted and all the medical prescriptions were reviewed for discontinuation of FDC during follow-up of the outpatient clinic visit. FDC discontinuation was defined as any discontinuation or splitting of the FDC treatment. However, a change in titration with the same FDC formula or the addition of any other medication concomitantly with the FDC was not considered to be a discontinuation. During review of the EMR of all the patients, CV events were defined as the composite of admission for controlling worsening heart failure or diabetes, stroke, ischaemic heart disease, and CV death. All events were evaluated and updated by reviewing the EMR or through telephone contact by a clinical physician. The follow-up duration was obtained from the index date of first prescribing FDC therapy to the occurrence of an event or last clinic visit (censored due to loss to follow-up). Follow-up completeness regarding the discontinuation of FDC and the CV events were $94.5 \%$ and $89.7 \%$, respectively, which were obtained by the index devised by Clark et al. ${ }^{12}$ The study patients were followed for a mean of $2.8 \pm 2.4$ years after the index date with respect to the CV events.

\section{Statistics}

Continuous variables are presented as mean \pm SD in cases of normal distribution and categorical variables as a number with the percentage. The 10-year ASCVD risk score for each patient was calculated using the pooled cohort equations provided by the ACC/AHA. Conventional CV risk scores were calculated for each patient by assigning one point to each risk and classifying the patients into tertiles according to the 20 risk factors (lower tertile $\leq 4,5 \leq$ mid tertile $\leq 7$, and upper tertile $\geq 8$ ). The 20 conventional risk factors were compared using the $\chi^{2}$ 
Table 1 Baseline characteristics of whole study populations according to the discontinuation of fixed-dose combination therapy

\begin{tabular}{|c|c|c|c|c|}
\hline & & Fixed-dose combina & & \\
\hline & Total patients $(n=502)$ & Continuing $(n=299)$ & Discontinued $(n=203)$ & $\mathrm{p}$ Value \\
\hline Age, years & $72.9 \pm 10.6$ & $72.3 \pm 10.3$ & $73.8 \pm 10.9$ & 0.119 \\
\hline Male, n (\%) & $202(40.2)$ & $118(39.5)$ & $84(41.4)$ & 0.711 \\
\hline Height, $\mathrm{cm}$ & $159.1 \pm 9.0$ & $158.8 \pm 9.1$ & $159.7 \pm 8.7$ & 0.259 \\
\hline Weight, kg & $65.4 \pm 9.5$ & $61.3 \pm 9.5$ & $62.1 \pm 9.1$ & $<0.001$ \\
\hline Body mass index, $\mathrm{kg} / \mathrm{m}^{2}$ & $25.3 \pm 3.4$ & $25.9 \pm 3.3$ & $24.3 \pm 3.3$ & $<0.001$ \\
\hline Systolic blood pressure, $\mathrm{mmHg}$ & $141.4 \pm 23.3$ & $144.3 \pm 22.1$ & $137.7 \pm 19.8$ & $<0.001$ \\
\hline Total cholesterol, mg/dL & $176.0 \pm 40.1$ & $181.0 \pm 39.4$ & $168.9 \pm 40.1$ & 0.001 \\
\hline Triglycerides, mg/dL & $131.0 \pm 71.9$ & $139.7 \pm 76.1$ & $118.5 \pm 63.5$ & 0.001 \\
\hline HDL cholesterol, mg/dL & $49.1 \pm 11.7$ & $48.7 \pm 12.1$ & $49.6 \pm 11.1$ & 0.407 \\
\hline LDL cholesterol, mg/dL & $105.4 \pm 33.3$ & $109.0 \pm 32.8$ & $100.0 \pm 31.8$ & 0.001 \\
\hline Fasting glucose, mg/dL & $112.3 \pm 35.4$ & $116.0 \pm 34.5$ & $106.9 \pm 36.1$ & 0.005 \\
\hline Creatinine, mg/dL & $0.99 \pm 0.81$ & $0.98 \pm 0.63$ & $1.03 \pm 0.99$ & 0.533 \\
\hline Smoking, n (\%) & $112(22.3)$ & $89(29.8)$ & $23(11.3)$ & $<0.001$ \\
\hline Alcohol intake, $\mathrm{n}(\%)$ & $134(26.7)$ & $114(38.1)$ & $20(9.8)$ & $<0.001$ \\
\hline Clinical history & & & & \\
\hline Heart failure, n (\%) & $52(10.4)$ & $47(15.7)$ & $5(2.5)$ & $<0.001$ \\
\hline Dyslipidaemia, n (\%) & $255(50.8)$ & $182(60.9)$ & $73(36.0)$ & $<0.001$ \\
\hline Diabetes mellitus, $\mathrm{n}(\%)$ & $141(28.1)$ & $94(31.4)$ & $47(23.2)$ & $<0.001$ \\
\hline Hypertension, $\mathrm{n}(\%)$ & $457(91.0)$ & $281(94.0)$ & $176(86.7)$ & 0.007 \\
\hline Ischaemic heart disease, $n(\%)$ & $172(34.3)$ & $126(42.1)$ & $46(22.7)$ & $<0.001$ \\
\hline Stroke, $n(\%)$ & $59(11.8)$ & $48(16.1)$ & $11(5.4)$ & $<0.001$ \\
\hline Peripheral vascular disease, $\mathrm{n}(\%)$ & $45(9.0)$ & $39(13.0)$ & $6(3.0)$ & $<0.001$ \\
\hline Renal replacement treatment, $\mathrm{n}(\%)$ & $10(2.0)$ & $5(1.7)$ & $5(2.5)$ & 0.535 \\
\hline Family history of CHD, $\mathrm{n}(\%)$ & $12(2.4)$ & $12(4.0)$ & $0(0.0)$ & 0.002 \\
\hline Total number of risk factors* & $6.47 \pm 2.83$ & $7.61 \pm 2.7$ & $4.80 \pm 2.2$ & $<0.001$ \\
\hline ASCVD risk scores & $28.8 \pm 17.6$ & $31.5 \pm 18.5$ & $24.8 \pm 15.4$ & $<0.001$ \\
\hline
\end{tabular}

${ }^{*}$ Conventional risk factors include age, body mass index, systolic blood pressure, total cholesterol, triglycerides, HDL cholesterol, LDL cholesterol, fasting glucose, creatinine, smoking, alcohol, heart failure, dyslipidaemia, diabetes mellitus, hypertension, ischaemic heart disease, stroke, peripheral vascular disease, chronic renal replacement therapy, and family history of premature coronary heart disease.

ASCVD, atherosclerotic cardiovascular disease; CHD, coronary heart disease; HDL, high-density lipoprotein; LDL, low-density lipoprotein

test for categorical variables, and one-way analysis of variance was performed for continuous variables among the three tertiles. Both the continuation of FDC and event-free survival curves were constructed to evaluate the adherence of FDC and the CV outcomes, respectively, using the Kaplan-Meier method and compared using the log-rank (Mantle-Cox) test. The significant variables $(\mathrm{p}<0.05)$ in the univariate Cox analysis were included in a multivariable analysis. The Cox proportional hazard regression model was performed to obtain the HR with 95\% CI for the discontinuation of FDC and the adverse CV events. The statistical analyses were performed using the Statistical Package for Social Science version 13.0 (SPSS Inc, Chicago, IL, USA). A value of $\mathrm{p}<0.05$ was considered to be statistically significant.

\section{RESULTS}

\section{Baseline cardiovascular risk factors of whole study patients}

A total of 502 patients (40\% male, mean age of 73 years) were analysed, and the types of prescribed FDCs were as follows: CCB plus ARB $(n=254,50.6 \%)$; CCB plus statins $(n=145,28.9 \%)$; and ARB plus diuretics $(n=103,20.5 \%)$. There were 203 discontinuations (40.4\%) of FDC therapy during a mean follow-up period of $2.8 \pm 2.4$ years (table 1 ). The FDC-discontinued patients showed more favourable baseline characteristics and laboratory results compared with the FDC-continuing patients. The prevalence of medical illness and family history of premature coronary heart disease were less frequently reported in the FDC-discontinued patients. The review of conventional risk factors and the ASCVD risk score revealed a small numbers of risk factors and low 10-year predicted risk scores, respectively, in the FDC-discontinued group.

\section{Association between maintenance of FDC and risk factors}

The 10-year ASCVD risk scores were lower in the FDC-discontinued group, irrespective of event occurrence (table 2). These findings were also consistent in the case of using conventional risk factors. Compared with the other tertiles of the number of conventional risk factors, the patients in the lower tertile did not maintain their FDC prescription and frequently split their FDC into multiple-drug prescriptions. Furthermore, the upper tertile showed significantly more events reported in the FDC-discontinued group compared with the respective lower and mid tertile groups.

Adverse CV events occurred more frequently in the discontinued group; however, the discontinued group had a smaller number of risk factors, even in patients experiencing CV events (figure 2). In the patients who discontinued their FDC, the mean number of risk factors was 6.0. The likelihood of maintaining or continuing FDC was significantly higher in patients with $>6$ risk factors compared with those having $\leq 6$ risk factors. Together with the 10-year ASCVD risk score, the small number of risk factors significantly contributed to discontinuing FDC therapy on univariate and multivariate analysis (table 3 ). The lower tertile group failed to continue FDC more easily than the upper or mid tertile groups in the early phase of treatment (figure $3 \mathrm{~A}$ ). 
Table 2 Association between fixed-dose combination discontinuation and risk scores, numbers or events

\begin{tabular}{|c|c|c|c|c|c|c|c|}
\hline & \multicolumn{3}{|c|}{ ASCVD risk scores, (\%) } & \multicolumn{4}{|c|}{ Events/total, n (\%) } \\
\hline & \multirow{2}{*}{$\begin{array}{l}\text { Events } \\
(+)(n=57)\end{array}$} & \multirow{2}{*}{$\begin{array}{l}\text { Events (-) } \\
(n=445)\end{array}$} & \multirow[b]{2}{*}{$\mathrm{p}$ Value } & \multicolumn{3}{|c|}{ Tertiles of the number of conventional risk factors* } & \multirow[b]{2}{*}{$\mathrm{p}$ Value } \\
\hline & & & & Lower $(n=157)$ & $\operatorname{Mid}(n=156)$ & Upper $(n=189)$ & \\
\hline FDC discontinued & $33.5 \pm 15.8$ & $23.3 \pm 14.9$ & 0.001 & $12 / 113(10.6)$ & $8 / 63(12.7)$ & $10 / 27(37.0)$ & 0.002 \\
\hline p Value & 0.033 & $<0.001$ & & 0.021 & 0.431 & 0.002 & \\
\hline
\end{tabular}

\section{Adverse events during follow-up}

During the study period, a total of $57 \mathrm{CV}$ events (11.4\%) occurred: 13 (2.6\%) episodes of heart failure, $16(3.2 \%)$ ischaemic heart disease events, 12 (2.4\%) strokes, 7 (1.4\%) episodes of worsening diabetes, and $9(1.8 \%) \mathrm{CV}$ deaths. No significant difference was found in the occurrence of $\mathrm{CV}$ events according to discontinuation of FDC (30 events (14.8\%) in the FDC-discontinued patients vs 27 (9.1\%) in the FDC-continued patients, $\mathrm{p}=0.062)$. However, in the Kaplan-Meier analysis, the patients who discontinued their FDC showed an unfavourable prognosis throughout the follow-up period (figure 3B). Both discontinuing the FDC and high ASCVD risk score were significantly associated with worse CV outcomes, although the upper tertile of the number of risk factors influenced the occurrence of adverse outcomes only in univariate analysis (table 4). The patients who discontinued their FDC therapy had nearly an eightfold greater risk of experiencing an adverse $\mathrm{CV}$ event.

\section{DISCUSSION}

Recently, the frequency of FDC prescriptions has increased to encourage medication compliance. ${ }^{1246}$ This is because FDC therapy is convenient and reduces the total pill count. Despite the advantages of FDC agents, they can be inflexible if dosing titration is required but the appropriate FDC dosage is unavailable. ${ }^{910}$ These findings might result in a high frequency of FDC discontinuation, and most of it could happen within 1 year, particularly in those with low ASCVD risk scores. However, it is still unclear why FDC discontinuation is more frequent than expected, despite its convenience. Our study revealed that two aspects should be considered: the patients' perception regarding

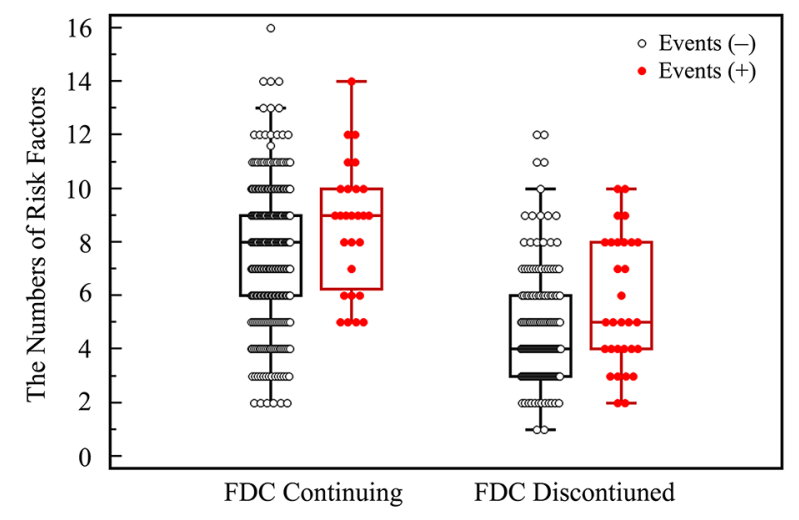

Figure 2 Association between the number of risk factors and fixeddose combination (FDC) discontinuation or cardiovascular events. Compared with the FDC-continuing group, the FDC-discontinued group has a small number of risk factors, irrespective of event occurrence.
FDC and disease severity, and the appropriate subset of patients to whom FDC should be prescribed.

The patients' knowledge of their disease underlies the maintenance of and adherence to FDC therapy. ${ }^{13}{ }^{14}$ Therefore, it is reasonable to presume that those patients with many risks and serious conditions have a high level of disease insight, and these high-risk patients would be more likely to adhere to their FDC regimen to improve or enhance their health status. ${ }^{15}$ However, some low risk patients believe that discontinuation of FDC would not contribute to immediate or early discomfort and may be ignorant of the importance of long-term medication within the CV disease spectrum. This belief would deem their disorders trivial and lead to poor compliance and FDC discontinuation. ${ }^{15-17}$ Conversely, strong adherence results in a good response of blood pressure control and a low frequency of adverse drug reactions..$^{18} 19$

The experience of a serious illness, per se, would also be strongly associated with adherence. Although patients who had more outpatient visits, underwent an interventional procedure or surgical operation, and had been hospitalised showed better adherence than those who had not, all of these experiences are usually considered as either high risk or a vulnerable state; thus, the compliance of these patients is critical to prevent their condition from deteriorating. ${ }^{2021}$ The majority of high-risk patients are willing to have good compliance even if they are only being treated for hypertension or hypercholesterolaemia. Interestingly, in the current study, nearly half of the patients in the lower tertile failed to continue FDC therapy within 1 year, while the upper tertile showed good adherence. Therefore, in regard to the enhancement of adherence, assertive encouragement or insight of FDC would be required in the early stage of treatment to prevent dropout.

There were limited data about the usefulness of FDC prescriptions regarding prevention of $\mathrm{CV}$ events. ${ }^{1-356}$ In the current study, a high rate of FDC discontinuation was observed in the low and mid tertile, or low ASCVD risk score group. According to the UMPIRE study, the adherence outcomes regarding blood pressure and cholesterol were significantly favourable in patients with a high estimated CV risk ( $>15 \%$ of 5 -year CV risk) and smokers. ${ }^{1}$ Those results would suggest the importance of FDC in high-risk patients in terms of preventing CVD. ${ }^{1-3}$ Those were in accordance with our results and could be explained by the simplicity of a single polypill and the strong motivation in established or high risk patients to stay healthy. ${ }^{22}$ Therefore, in patients with established CV diseases or at high risk, a clear benefit of FDC was observed as indicated in cases of clustering risk factors such as dyslipidaemia, hypertension, and diabetes. ${ }^{323}$ Among the high-risk patients, the benefit gain would increase by controlling the outcomes of many clustering risk factors using a simple prescription. Although the effect of FDC on CV events 
Table 3 Univariable and multivariable Cox analysis for discontinuation of fixed-dose combination therapy

\begin{tabular}{|c|c|c|c|c|}
\hline & \multicolumn{2}{|l|}{ Univariable } & \multicolumn{2}{|l|}{ Multivariable } \\
\hline & HR $(95 \% \mathrm{Cl})$ & $p$ Value & HR $(95 \% \mathrm{Cl})$ & $\mathrm{p}$ Value \\
\hline \multicolumn{5}{|l|}{ Types of FDC } \\
\hline CCB and statin & 1 (Reference) & - & - & \\
\hline$C C B$ and $A R B$ & 1.009 (0.699 to 1.458$)$ & 0.960 & & \\
\hline ARB and diuretics & $0.747(0.527$ to 1.059$)$ & 0.101 & & \\
\hline ASCVD risk scores, \% & 0.981 (0.972 to 0.989$)$ & $<0.001$ & $0.984(0.975$ to 0.990$)$ & 0.003 \\
\hline \multicolumn{5}{|c|}{ Tertiles of the numbers of conventional risk factors* } \\
\hline Lower tertile & 1 (Reference) & - & 1 (Reference) & - \\
\hline Mid tertile & $0.430(0.316$ to 0.586$)$ & $<0.001$ & 0.432 (0.314 to 0.593$)$ & $<0.001$ \\
\hline Upper tertile & $0.113(0.174$ to 0.173$)$ & $<0.001$ & $0.114(0.072$ to 0.179$)$ & $<0.001$ \\
\hline
\end{tabular}

was not obtained because of the low incidence of events (11\%) in the Cochrane database and a relatively short follow-up period (2 years) in the UMPIRE trials, ${ }^{16}{ }^{24}$ both the physician's insight and efforts to control risk factors could affect the long-term CV prognosis in patients using FDC as shown in our study, which was designed for the intensive and broad control of clustering risk factors.

Although there was no significant association between the total number of risk factors and the development of CV events in the FDC-continuing group $(p=0.054)$, the discontinuation of FDC therapy was strongly related to CV events $(p=0.002)$. However, considering the limited data about FDC in realworld practice, it is not easy to determine to what extent FDC discontinuation would contribute to $\mathrm{CV}$ adverse outcomes. In the present analysis, FDC discontinuation appeared to be more important than other factors including ASCVD risk score or factors. This is because the maintenance of FDC therapy could modify or reduce risk factors, indicating improved clinical results. ${ }^{2}$ Thus, the FDC-discontinued patients would be exposed again to high CV risk including uncontrolled hypertension or unfavourable lipid profile over time, although they had low risk score or factors at the time of receiving their FDC prescription. This might frame a concept that FDC discontinuation could not control risk factors, particularly in modest- or high-risk patients. Besides, the patients who discontinued their FDC therapy (and may have required up or down titration with non-FDC drugs) were compelled to obtain much more complicated regimens, resulting in either low compliance or increased frequency of side effects.

Ultimately, not all patients are believed to require FDC therapy. This makes it crucial to select the most appropriate candidates. The strong relationship between discontinuing FDC and the occurrence of $\mathrm{CV}$ events was observed and was most profound in the upper tertile, which was compatible with the findings of the SPACE study; the definite benefit of FDC was consistently observed in high-risk patients regarding blood pressure and dyslipidaemia. ${ }^{2}$ The importance of FDC appeared to be significant in the patients with many risk factors. Another explanation is that it appears likely that patients either with few risk factors or without a diagnosis of a high-risk condition would not need a multipotent FDC. Therefore, the advantages of an FDC over a multi-pill regimen may be greater for high-risk patients or those who require a simple drug regimen, in addition to the definite advantage of reducing pill counts.
A

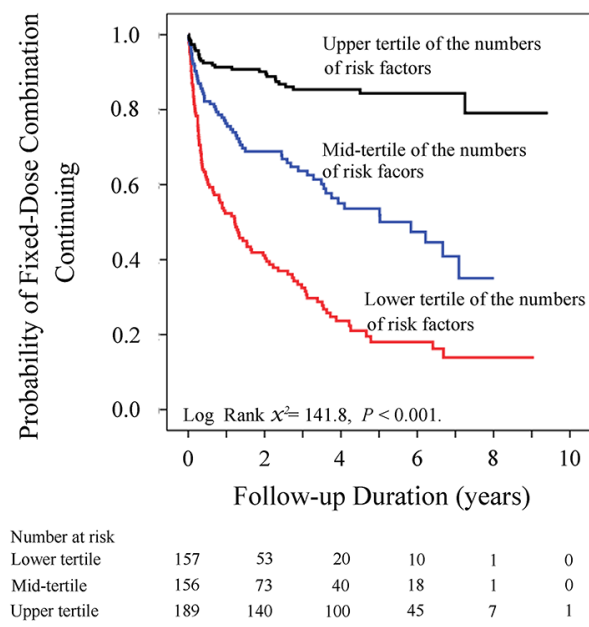

B

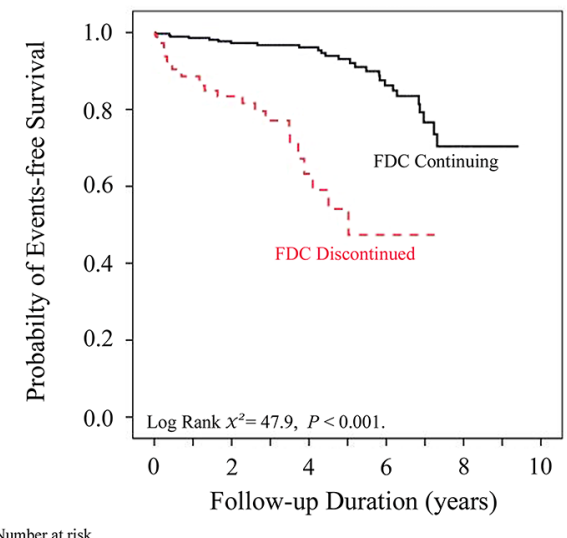

Number at risk

$\begin{array}{lrrrrrr}\text { FDC Discontinued } & 299 & 213 & 145 & 67 & 9 & 1\end{array}$

Figure 3 (A) Kaplan-Meier curve for continuation of the fixed-dose combination (FDC) according to tertiles of the numbers of risk factors. Nearly half of the patients in the lower tertile showed discontinuation of FDC within 1 year of prescription, whereas those in the upper tertile had good adherence of FDC. (B) Kaplan-Meier curve for the primary outcome of adverse events according to FDC continuation or discontinuation. 
Table 4 Univariable and multivariable Cox analysis for adverse events

\begin{tabular}{|c|c|c|c|c|}
\hline & \multicolumn{2}{|l|}{ Univariable } & \multicolumn{2}{|l|}{ Multivariable } \\
\hline & HR $(95 \% \mathrm{Cl})$ & $p$ Value & HR $(95 \% \mathrm{Cl})$ & $\mathrm{p}$ Value \\
\hline \multicolumn{5}{|l|}{ Types of FDC } \\
\hline CCB and statin & 1 (Reference) & - & - & \\
\hline$C C B$ and $A R B$ & 1.11 (0.55 to 2.28$)$ & 0.768 & & \\
\hline ARB and diuretics & $0.93(0.47$ to 1.82$)$ & 0.822 & & \\
\hline Discontinuation of FDC & 6.193 (3.504 to 10.94$)$ & $<0.001$ & 7.590 (4.042 to 14.26$)$ & $<0.001$ \\
\hline ASCVD risk scores, \% & $1.020(1.006$ to 1.035$)$ & 0.005 & 1.026 (1.008 to 1.045$)$ & 0.017 \\
\hline \multicolumn{5}{|c|}{ Tertiles of the numbers of conventional risk factors* } \\
\hline Lower tertile & 1 (Reference) & - & 1 (Reference) & - \\
\hline Mid tertile & $1.382(0.630$ to 3.021$)$ & 0.420 & 1.122 (0.516 to 2.438$)$ & 0.772 \\
\hline Upper tertile & 2.190 (1.083 to 4.451$)$ & 0.030 & $1.527(0.643$ to 3.629$)$ & 0.338 \\
\hline
\end{tabular}

${ }^{*}$ Conventional risk factors include age, body mass index, systolic blood pressure, total cholesterol, triglycerides, HDL cholesterol, LDL cholesterol, fasting glucose, creatinine, smoking, alcohol, heart failure, dyslipidaemia, diabetes mellitus, hypertension, ischaemic heart disease, stroke, peripheral vascular disease, chronic renal replacement therapy and family history of premature coronary heart disease.

ARB, angiotensin receptor blocker; ASCVD, atherosclerotic cardiovascular disease; CCB, calcium channel blocker; FDC, fixed-dose combination; lower tertile $\leq 4$ risk numbers; $5 \leq$ mid-tertile $\leq 7$ risk numbers; upper tertile $\geq 8$ risk numbers.

There were several limitations to the present study. First, this was a retrospective study and the adherence to FDC therapy could not be evaluated using objective tools or programmes such as the Morisky Medication Adherence Scale. In cases of discontinued FDC, the reason for discontinuation could not be obtained for the entire patient cohort. In addition, the frequency of clinic visits and the socioeconomic status of the cohort were not assessed. These could cause self-selection bias, resulting in a biased sample. This is because a patient's compliance, motivation, and prior medical illness experience are closely related to FDC continuation or clinical outcomes. Second, FDC of dual anti-platelet therapy (DAPT) or non-CV drugs were excluded. While the FDC of DAPT is widely used for coronary artery disease, the discontinuation or splitting of DAPT into monotherapy could be suggested for preventing the complication of bleeding after 6-12 months of coronary stents. Those may cause the important distortion or concerns for analysis of prescription or response of FDC. Third, the inclusion of individuals with prevalent ischaemic heart disease in the ACC/AHA pooled cohort equation calculation may lead to erroneous risk estimation. This would affect the underestimation of the ASCVD risk score. Last, we recognised that there were also limitations

\section{Key messages}

\section{What is already known about this subject?}

Fixed-dose combination (FDC) drugs are increasingly prescribed to enhance adherence, but discontinuation of FDC is also common in the real-world setting.

\section{What does this study add?}

Patients who discontinued their FDC drugs had lower 10-year atherosclerotic disease (ASCVD) risk scores or a smaller number of risk factors. Nearly half of the patients with $<4$ risk factors discontinued their FDC medication within 1 year of prescription. The discontinuation of FDC therapy and high ASCVD risk scores were related to cardiovascular (CV) events.

\section{How might this impact on clinical practice?}

Despite the advantage of FDC therapy, clinicians need to bear in mind that the discontinuation of FDC drugs in patients at high ASCVD risk may contribute to the occurrence of adverse CV events. regarding the statistical analysis. A restricted cubic spline curve was performed because the relationship between the discontinuation of FDC and the number of risk factors was non-linear and showed an exponential distribution. Also, it was difficult to analyse all the risk factors and to consider adding all of them into a multivariable analysis. Therefore, each risk factor was dichotomised and risk scores were calculated.

In conclusion, the high frequency of FDC discontinuation was found in the lower and mid tertile groups, particularly during the early phase of treatment. With respect to the CV outcomes, the FDC-discontinued patients at high ASCVD risk score showed an unfavourable prognosis; thus it would be necessary to encourage FDC adherence in these patients.

Acknowledgements The authors would like to acknowledge M-Y Kim, RN, and $\mathrm{J}-\mathrm{H}$ Jeon, RN, for their significant contribution to this study.

Contributors Study design: HK; data collection: HJY, HSP, YKC; data analysis: HK, CWN, SH, SHH; manuscript writing: HK; manuscript approval: YNK, KBK.

Competing interests None declared.

Ethics approval IRB, Keimyung University Dongsan Medical Center.

Provenance and peer review Not commissioned; externally peer reviewed.

Data sharing statement Nothing.

(c) Article author(s) (or their employer(s) unless otherwise stated in the text of the article) 2017. All rights reserved. No commercial use is permitted unless otherwise expressly granted.

\section{REFERENCES}

1 Thom S, Poulter N, Field J, et al. Effects of a fixed-dose combination strategy on adherence and risk factors in patients with or at high risk of CVD: the UMPIRE randomized clinical trial. JAMA 2013;310:918-29.

2 Webster R, Patel A, Selak V, et al. Effectiveness of fixed dose combination medication ('polypills') compared with usual care in patients with cardiovascular disease or at high risk: a prospective, individual patient data meta-analysis of 3140 patients in six countries. Int J Cardiol 2016;205:147-56.

3 Lafeber M, Webster R, Visseren FLj, et al. Estimated cardiovascular relative risk reduction from fixed-dose combination pill (polypill) treatment in a wide range of patients with a moderate risk of cardiovascular disease. Eur J Prev Cardiol 2016;23:1289-97.

4 Bryant L, Martini N, Chan J, et al. Could the polypill improve adherence? The patient perspective. J Prim Health Care 2013;5:28-35.

5 Patel A, Cass A, Peiris D, et al. A pragmatic randomized trial of a polypill-based strategy to improve use of indicated preventive treatments in people at high cardiovascular disease risk. Eur J Prev Cardiol 2015;22:920-30.

6 Bahiru E, de Cates AN, Farr MR, et al. Fixed-dose combination therapy for the prevention of atherosclerotic cardiovascular diseases. Cochrane Database Syst Rev 2017;3:CD009868. 
7 Bosworth HB, Granger BB, Mendys P, et al. Medication adherence: a call for action. Am Heart J 2011;162:412-24.

8 Yusuf S, Attaran A, Bosch J, et al. Combination pharmacotherapy to prevent cardiovascular disease: present status and challenges. Eur Heart J 2014;35:353-64.

9 Webster R, Rodgers A. Polypill treatments for cardiovascular diseases. Expert Opin Drug Deliv 2016;13:1-6.

10 Angeli F, Reboldi G, Mazzotta G, et al. Fixed-dose combination therapy in hypertension: cons. High Blood Press Cardiovasc Prev 2012;19:51-4.

11 Wertheimer Al. The economics of polypharmacology: fixed dose combinations and drug cocktails. Curr Med Chem 2013;20:1635-8.

12 Clark TG, Altman DG, De Stavola BL. Quantification of the completeness of followup. Lancet 2002;359:1309-10.

13 Christensen AJ, Howren MB, Hillis SL, et al. Patient and physician beliefs about control over health: association of symmetrical beliefs with medication regimen adherence. J Gen Intern Med 2010:25:397-402.

14 Kronish IM, Ye S. Adherence to cardiovascular medications: lessons learned and future directions. Prog Cardiovasc Dis 2013:55:590-600.

15 Murray KA, Murphy DJ, Clements S-J, et al. Comparison of uptake and predictors of adherence in primary and secondary prevention of cardiovascular disease in a community-based cardiovascular prevention programme (MyAction Westminster). J Public Health 2014;36:644-50.

16 Halava H, Korhonen MJ, Huupponen R, et al. Lifestyle factors as predictors of nonadherence to statin therapy among patients with and without cardiovascular comorbidities. CMAJ 2014;186:E449-56.
17 Anderson DR, Emery CF. Irrational health beliefs predict adherence to cardiac rehabilitation: a pilot study. Health Psychol 2014;33:1614-7.

18 Bramlage $\mathrm{P}$, Ketelhut R, Fronk EM, et al. Clinical impact of patient adherence to a fixed-dose combination of olmesartan, amlodipine and hydrochlorothiazide. Clin Drug Investig 2014;34:403-11.

19 Mazzaglia G, Ambrosioni E, Alacqua M, et al. Adherence to antihypertensive medications and cardiovascular morbidity among newly diagnosed hypertensive patients. Circulation 2009;120:1598-605.

20 Oosterom-Calo R, van Ballegooijen AJ, Terwee CB, et al. Determinants of adherence to heart failure medication: a systematic literature review. Heart Fail Rev 2013;18:409-27.

21 Cholowski K, Cantwell R. Predictors of medication compliance among older heart failure patients. Int J Older People Nurs 2007;2:250-62.

22 Wood F, Salam A, Singh K, et al. Process evaluation of the impact and acceptability of a polypill for prevention of cardiovascular disease. BMJ Open 2015; 5:e008018.

23 Lafeber $\mathrm{M}$, Spiering W, van der Graaf $\mathrm{Y}$, et al. The combined use of aspirin, a statin, and blood pressure-lowering agents (polypill components) and the risk of vascular morbidity and mortality in patients with coronary artery disease. Am Heart $J$ 2013:166:282-9

24 Castellano JM, Sanz G, Peñalvo JL, et al. A polypill strategy to improve adherence: results from the FOCUS project. J Am Coll Cardiol 2014;64:2071-82. 